\title{
National Institute of Neurological Disorders and Stroke support for brain-machine interface technology
}

\author{
Joseph J. Pancrazio, Ph.D. \\ National Institute of Neurological Disorders and Stroke, National Institutes of Health, Rockville, Maryland
}

\begin{abstract}
Brain-machine interfaces (BMIs) offer the promise of restoring communication, enabling control of assistive devices, and allowing volitional control of extremities in paralyzed individuals. Working in multidisciplinary teams, neurosurgeons can play an invaluable role in the design, development, and demonstration of novel BMI technology. At the National Institutes of Health, the National Institute of Neurological Disorders and Stroke has a long history of supporting neural engineering and prosthetics efforts including BMI, and these research opportunities continue today. The author provides a brief overview of the opportunities and programs currently available to support BMI projects. (DOI: 10.3171/2009.3.FOCUS0989)
\end{abstract}

\section{KeY Words - brain-machine interface $\bullet$ National Institute of Neurological Disorders and Stroke}

$\mathrm{B}$ RAIN-MACHINE interfaces provide a pathway for communication between the brain and devices that are either external or internal to the patient. In bypassing regions of the nervous system damaged as a result of trauma or disease, BMIs have significant implications for the restoration of communication for lockedin patients, volitional control of paralyzed limbs through the activation of implanted neuromuscular stimulation systems, and perhaps sensorimotor function in prosthetic limbs. ${ }^{4}$ Such interfaces are being realized through cooperative multidisciplinary efforts among basic neuroscientists, biomedical engineers, and neurosurgeons. The foundation of BMIs is firmly embedded in basic neuroscience, which aims to elucidate both how the brain controls movement and the capacity of brain circuitry to undergo adaptation. By leveraging advances in the microelectronics industry and computer engineering, the BMI field has capitalized on precisely microfabricated neural interfaces ${ }^{8}$ and, more importantly, high-speed data acquisition and processing to enable real-time use.

In general, there are 3 classes of BMIs that vary with respect to invasiveness and are widely seen to differ with respect to data rates. The least invasive approach, electroencephalography, is frequently used in clinical neurology for diagnostic applications and is based on decoding scalp-derived potentials and rhythms. ${ }^{1}$ While electroencephalography potentials consist of the cumulative spike activity of many localized and similarly oriented neu-

\footnotetext{
Abbreviations used in this paper: $\mathrm{BMI}=$ brain-machine interface; $\mathrm{NIH}=$ National Institutes of Health; NINDS = National Institute of Neurological Disorders and Stroke; SBIR = Small Business Innovation Research; STTR = Small Business Technology Transfer.
}

rons, invasive BMIs consisting of microelectrode arrays simultaneously monitor spike activity from populations of distinguished units, each representing putative individual neurons. ${ }^{6}$ Electrocorticography, which relies on subdural recording grids to record field potentials, has emerged as an intermediate approach that may offer a compromise with respect to invasiveness but with high performance. ${ }^{7}$ Although these devices hold significant promise for relieving the burden of neurological disease and injury, the field is currently at a nascent stage requiring cultivation through federal support. The purpose of this paper is to provide a brief overview of how the NINDS has supported BMI research in the past and to describe opportunities and programs currently available to support BMI projects.

\section{Neural Prosthesis Program}

The initial scientific driving force for developing devices that interface with the nervous system emerged in the late 1950s with the first detailed description of the effects of direct electrical stimulation of the auditory nerve in the context of deafness, leading to perception. ${ }^{3}$ In 1968 Brindley and Lewin ${ }^{2}$ described the visual percepts of a blind person who had undergone implantation of an electrode array on the surface of the occipital cortex. Their work and other related efforts spawned a series of questions concerning electrode robustness and stability, tissue-device compatibility, fundamental mechanisms of neural excitation by electrical stimulation, and principles of information encoding and transfer within the nervous system. Given the targeted and interdisciplinary nature of these initial questions, the NIH Neural Prosthesis Pro- 
gram $^{5}$ was launched in 1970 and achieved a number of accomplishments under the leadership of Drs. K. Frank, T. Hambrecht, and W. Heetderks. Among the most significant achievements has been the cochlear implant, a neural interface that provides a sense of sound to profoundly deaf or severely hard-of-hearing individuals by processing sounds from the environment and directly stimulating the auditory nerve.

\section{Support for BMI Research and Development}

More recently, projects within the Neural Prosthesis Program have been able to utilize grant programs to support BMI research and development. In 1997 the NIH recognized the potential contributions of bioengineering to the mission of relieving the burden of disease, and it implemented a set of related funding opportunities. A key feature of these programs is that each allows the pursuit of milestone-oriented, rather than hypothesis-driven, aims-goals consistent with engineering design and development activities. Current bioengineering program announcements support neural engineering activities including BMI research: Exploratory Bioengineering Research Grants (R21, PA-06-418): 2-year, high-risk, high-return, early-stage bioengineering projects; Bioengineering Research Grants (R01, PA-07-279): 4- to 5-year projects intended to support continuing studies in bioengineering; and Bioengineering Research Partnerships (R01, PAR07-352): 5-year projects pursued through a multidisciplinary research team that applies an integrative systems approach to biomedical problem solving.

The proof-of-concept demonstration of neurotechnology presents opportunities in the private sector. The NINDS supports small business activities in neurotechnology through the parent SBIR and STTR programs listed in the NIH omnibus (http://grants.nih.gov/grants/ funding/sbir.htm). In addition, the NINDS calls for SBIR and STTR research pertaining to BMI through the Neurotechnology Research, Development, and Enhancement program (SBIR Phase I and II: PA-07-389 and STTR Phase I and II: PA-07-390). To bridge the gap between the bench and the clinic, the NINDS is offering new program announcements for cooperative agreement-based grants encouraging translational and pilot clinical studies for neural prosthetics: Advanced Neural Prosthetics Research and Development (U01, PA-09-063): 4- to 5-year projects; and Advanced Neural Prosthetics Research and Development (U44, PA-09-064): 3-5 fast-track or Phase II SBIR projects.

These programs provide funding for milestone-driven projects for the design, development, and demonstration of clinically useful neural prosthetic devices including BMIs. Activities to be supported in these programs include the implementation of clinical prototype devices, preclinical safety and efficacy testing, design verification and validation activities, the pursuit of regulatory approval for clinical study, and proof-of-concept or pilot clinical studies. With these cooperative agreement mechanisms, there is significant participation of NIH staff in the evaluation of milestones and coordination with other neural engineering activities supported by the NIH or other federal agencies.
The commitment of the NIH in fostering neurotechnology-related research extends beyond the development of programs. In 2007 the NIH Center for Scientific Review established a new Integrated Review Group called Emerging Technologies and Training in Neurosciences (ETTN). Within this group, The Neurotechnology Study Section considers applications seeking to develop and use computational, informatics, imaging, biophysical, and bioengineering approaches for neuroscience. This Section provides a review forum for consistent and expert review of proposals for BMIs and neuroprosthetic devices.

\section{Conclusions}

In the BMI field, research teams that engage neurosurgeons as full partners in their endeavors are poised to have significant impact. Obviously, neurosurgeons have a critical role in realizing clinical demonstrations of this nascent technology, but their contributions can also be made earlier in the project through the design control process (design reviews, design verification, and design validation), a key activity for regulatory approval.

\section{Disclaimer}

The author reports no conflict of interest concerning the materials or methods used in this study or the findings specified in this paper.

\section{Acknowledgment}

The views expressed here are the author's and do not represent those of the NIH or the US government. No official support or endorsement by the NIH is intended or should be inferred.

\section{References}

1. Birbaumer N, Cohen LG: Brain-computer interfaces: communication and restoration of movement in paralysis. J Physiol 579:621-636, 2007

2. Brindley GS, Lewin WS: Sensations produced by electrical stimulation of visual cortex. J Physiol 196:479-493, 1968

3. Djourno A, Eyries C: Prothèse auditive par excitation électrique à distance du nerf sensoriel à l'aide d'un bobinage inclus à demeure. Presse Med 65:1417, 1957 (Fr)

4. Fagg AH, Hatsopoulos NG, de Lafuente V, Moxon KA, Nemati S, Rebesco JM, et al: Biomimetic brain machine interfaces for the control of movement. J Neurosci 27:11842-11846, 2007

5. Frank K, Hambrecht FT: Neural prostheses. Brain Behav Evol 14:7-9, 1977

6. Lebedev MA, Nicolelis MAL: Brain machine interfaces-past, present, and future. Trends Neurosci 29:536-546, 2006

7. Schalk G, Miller KJ, Anderson NR, Wilson JA, Smyth MD, Ojemann JG, et al: Two-dimensional movement control using electrocorticographic signals in humans. J Neural Eng 5:75-84, 2008

8. Wise KD, Sodagar AM, Yao Y, Gulari MN, Perlin GE, Najafi $\mathrm{K}$, et al: Microelectrodes, microelectronics, and implantable neural microsystems. Proc IEEE 96:1184-1202, 2008

Manuscript submitted March 15, 2009.

Accepted March 24, 2009.

Address correspondence to: Joseph J. Pancrazio, Ph.D., National Institute of Neurological Disorders and Stroke, National Institutes of Health, NSC 2205, 6001 Executive Boulevard, Rockville, Maryland 20892. email: pancrazj@ninds.nih.gov. 